\title{
Quitting Patterns and Predictors of Success Among Participants in a Tobacco Cessation Program Provided by Pharmacists in New Mexico
}

\author{
Xian Shen, MS; Amy Bachyrycz, PharmD; Joe R. Anderson, PharmD; \\ Dale Tinker, BA; and Dennis W. Raisch, PhD, MS, RPh
}

\section{ABSTRACT}

BACKGROUND: Tobacco use causes hundreds of thousands of deaths in the United States each year. Pharmacists are available in the community to provide tobacco cessation interventions. Between 2004 and 2010, the New Mexico Pharmaceutical Care Foundation (NMPCF) provided a pharmacistled tobacco cessation program to residents in New Mexico.

OBJECTIVES: To (a) obtain point prevalence quit rates at 1 month, 3 months, and 6 months follow-up for participants enrolled in the NMPCF program;

(b) differentiate between the quitting patterns of enrolled participants; and

(c) identify predictors associated with the quitting patterns.

METHODS: Seven-year data were combined for the pattern analysis. Four quitting patterns were defined, including immediate quitters, delayed quitters, once quitters, and never quitters. Multinomial logistic regression was used to identify characteristics of participants with different quitting patterns.

RESULTS: The analysis included 1,437 participants. The average point prevalence quit rate at 1 month, 3 months, and 6 months was $29.3 \%$, $23.3 \%$, and $18.0 \%$, respectively. Based on our definition for quitting patterns, the study sample consisted of $145(10.1 \%)$ immediate quitters, 113 (7.9\%) delayed quitters, $298(20.7 \%)$ once quitters, and $881(61.3 \%)$ never quitters. Multinomial logistic regression identified associations between quitting patterns and demographics, tobacco use and restrictions, baseline confidence in successful quitting, and pharmacotherapy aids used to quit. Relationships varied between quitting patterns.

CONCLUSIONS: The study findings showed that having community pharmacists provide smoking cessation interventions resulted in quitting success rates similar to other health care professionals, which ranged from $9.9 \%$ to $26.0 \%$. Since pharmacists are a widely available resource for their patients, managed care organizations may be able to improve the health, and avoid subsequent tobacco-related adverse health outcomes, of their members by implementing a program similar to the NMPCF Tobacco Cessation Program.

J Manag Care Pharm. 2014;20(6):579-87

Copyright $\odot 2014$, Academy of Managed Care Pharmacy. All rights reserved.

\section{What is already known about this subject}

Tobacco use is a major public health concern in the United States, causing hundreds of thousands of deaths annually.

Healthy People 2020 includes objectives for increasing tobacco screening and tobacco cessation counseling in health care settings by $10 \%$.

\section{What this study adds}

The study sample, consisting of 1,437 participants residing in rural or urban areas throughout New Mexico over 7 years, is larger than that of other pharmacist-led tobacco cessation programs previously reported in the literature.

The findings on the program quit rates suggest that having community pharmacists provide smoking cessation interventions results in quitting success rates similar to other health care professionals.

The multivariate analysis of quitting patterns provides insights into which individuals may have more difficulty achieving tobacco cessation and thus informs planning of future interventions.

The relationships between study variables (such as confidence to quit, type of tobacco used, nicotine dependence level, and pharmacotherapy aids) and quitting patterns can be used to design strategies for pharmacists to improve tobacco cessation rates among specific groups.

T he transtheoretical model of change (TTM) is a widely known theory for health behavior changes and has been applied to guide tobacco cessation interventions. It proposes that tobacco users move through 5 stages before they successfully achieve abstinence. The 5 stages are precontemplation (not thinking about quitting), contemplation (thinking about quitting), preparation (planning to quit in the next 30 days), action (quitting successfully for up to 6 months), and maintenance (no tobacco use for more than 6 months). ${ }^{1-3}$ Quitting tobacco is a difficult task with multiple barriers, including weight gain, craving for smoking, loss of the pleasure associated with smoking, stress and depression after quitting, and temptations from being around smokers. ${ }^{4,5}$ It is a process that usually is effective when interventions are tailored to individuals' specific stages and efforts are repeated for reinforcing treatment effects. Individuals in different stages might require different types of support and might return from advanced stages to earlier stages during the process. A pharmacist-assisted tobacco cessation program based in a Veterans Health Administration outpatient clinic in Montana showed an increase in 6-month success rates from $29.8 \%$ to $41.5 \%$ after incorporating TTM into the program. ${ }^{6}$ Specifically based on TTM, the program was designed to include 3 specific group counseling sessions that incorporated relevant educational 
information and behavioral strategies to move patients from contemplation to preparation (session 1: preparing to quit), from preparation to action (session 2: taking action), and from action to maintenance (session 3: maintaining abstinence). ${ }^{6}$ The success of the program indicates that integration of TTM can improve quit rates of tobacco cessation interventions.

The quit rates of previously reported pharmacist-assisted tobacco cessation programs in the United States range from 9.9\% to $26.0 \%$. $^{7-9}$ A meta-analysis reported in the Guidelines for Treating Tobacco Use and Dependence 2008 Update showed that the effectiveness of tobacco cessation programs delivered by different types of health care professionals was comparable. ${ }^{10}$ A study reported a $10.0 \%$ quit rate at 6 months among 465 participants who received tobacco cessation counseling with a primary care physician. ${ }^{11}$ Under a randomized clinical trial setting, a nurse-managed tobacco cessation program achieved a quit rate of $19.0 \%$ at 12 months. ${ }^{12}$ A database analysis of 1,477 patients within a private health plan who were prescribed with varenicline showed that 521 (35.3\%) patients ceased smoking at 12 months. ${ }^{13}$ While quit rates of various interventions have been reported previously, few studies have presented a longitudinal picture of patterns of postintervention smoking behaviors. ${ }^{14,15}$ In order to better understand the process of tobacco cessation and improve future interventions, quitting patterns and smokers' transitions in smoking status that emerge during a quitting attempt need to be evaluated.

In 2004, the New Mexico Board of Pharmacy granted pharmacists the authority to prescribe tobacco cessation products with an approved protocol. To support pharmacists' involvement in tobacco cessation and increase availability of tobacco cessation services to residents in New Mexico, the New Mexico Pharmaceutical Care Foundation (NMPCF) initiated a pharmacist-assisted tobacco cessation program in the same year. Participating pharmacists were reimbursed for providing the service through funding from the New Mexico (NM) Tobacco Use Prevention and Control Program (TUPAC). Between 2004 and 2010, 22 community pharmacies and 1 institutional Veterans Health Administration pharmacy in New Mexico participated in the program for at least 1 calendar year. In order to ensure and strengthen pharmacists' capability of counseling tobacco use cessation, interested pharmacists completed a comprehensive training program ( $\mathrm{Rx}$ for Change). ${ }^{16}$ The Rx for Change curriculum, which incorporates TTM components, has been disseminated to pharmacy schools throughout the United States and has obtained acceptance and positive feedback. ${ }^{17}$ The program included all necessary materials and supplies for providing tobacco cessation services for the NM TUPAC program. Training included the following: a description of the National Cancer Institute's 5 A's (i.e., Ask, Advise, Assess, Assist, Arrange), ${ }^{18}$ stages of behavior change, counseling techniques, the standard statewide-approved pharmacotherapy protocol, and data collection and reporting procedures. The pharmacotherapy protocol was approved by the NM Medical Board, the NM Board of Nursing, and the NM Board of Pharmacy. Following the protocol, pharmacists were allowed to prescribe products approved by the U.S. Food and Drug Administration for tobacco cessation independent of other practitioners. Pharmacists received continuing education credits for the training through the Accreditation Council for Pharmacy Education. Pharmacists in rural areas could complete the web-based Rx for Change tobacco cessation curriculum if they could not attend the in-person training sessions. Pharmacists were followed up 1 month after the training, either in person or by phone, to clarify and reinforce the concepts presented in the training. Participants for the tobacco cessation program were recruited by the participating pharmacists at each local pharmacy. The program was not advertised through purchased media, but press releases were provided to local media regarding the local pharmacy participation in the program. The NM TUPAC promoted a toll-free number (1-800-QUITNOW), which referred patients to the tobacco cessation program. The pharmacists had the option to promote the program locally through word of mouth. The pharmacists talked with patients when they purchased over-the-counter cessation products and informed them about the program and encouraged participation.

The program provided participants with pharmacotherapies, such as nicotine replacement therapy and varenicline, up to the value of $\$ 137.50$, and/or free counseling sessions with a pharmacist. Participants were not compensated financially for their participation.

Although the NMPCF program was discontinued in 2011 due to lack of funding, data were available to describe the success of a pharmacist-led tobacco cessation program in community settings as well as to identify factors associated with quitting patterns. The objectives of this study were (a) to obtain point prevalence quit rates at 1 month, 3 months, and 6 months follow-up for participants enrolled in the NMPCF program; (b) to differentiate between the quitting patterns of enrolled participants; and (c) to identify predictors associated with the quitting patterns.

\section{Methods}

Data from the NMPCF program between 2004 and 2010 were combined for analysis. Intake and follow-up questionnaires developed by the NM TUPAC program were adopted and administered by pharmacists. The intake questionnaire was administered to each participant at recruitment to obtain baseline information on tobacco use, nicotine dependence, previous quit attempts, work and home environments, confidence to quit, importance to quit, and demographic characteristics. The intake questionnaire took approximately 45-60 minutes to complete. Three follow-up sessions (1 month, 3 months, and 6 months) were scheduled to attain participants' status of tobacco use and provide additional counseling and support. The follow-up 


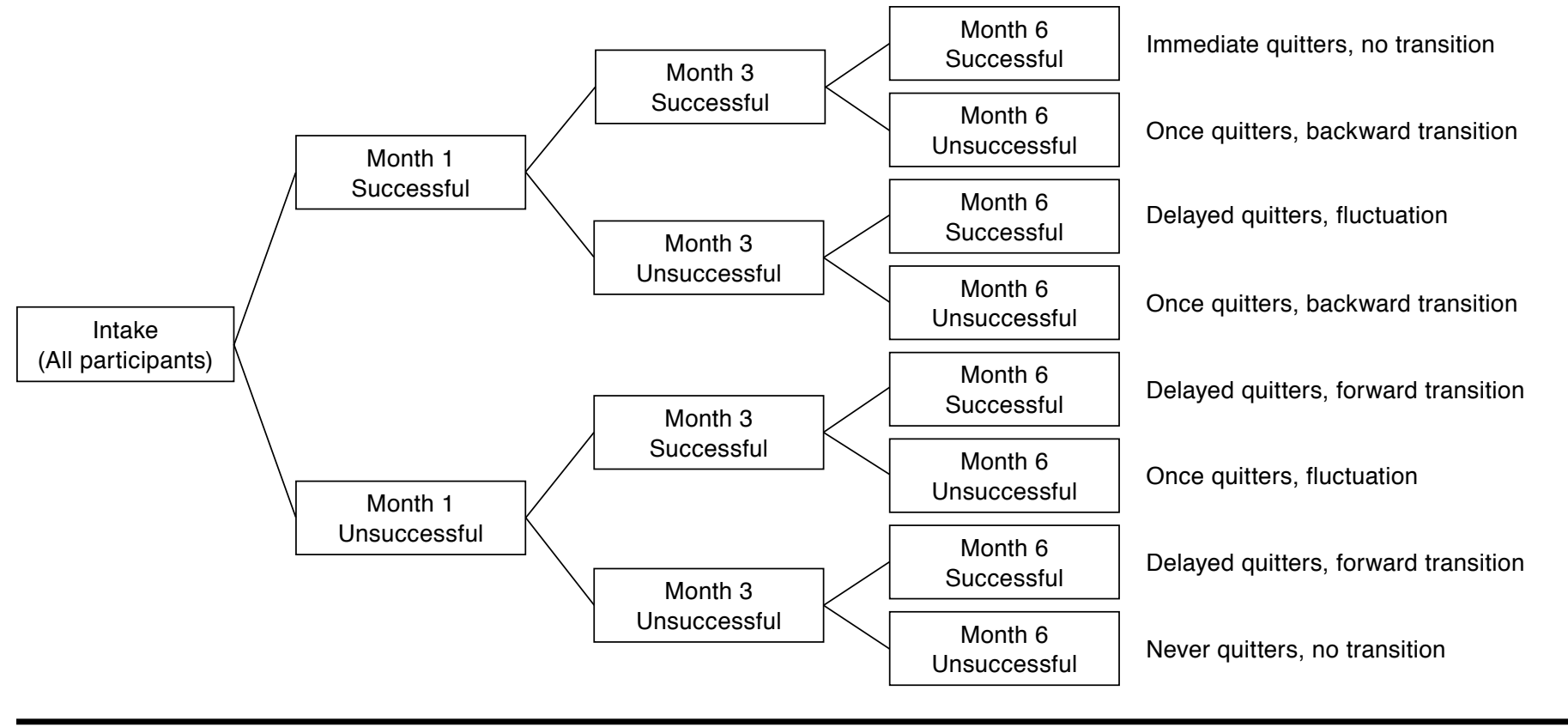

sessions were usually 15 minutes in person or by phone. Participants who responded to at least 1 of the 4 questionnaires (1 intake and 3 follow-up) were included for analysis.

For each follow-up time point, an average point prevalence quit rate across years, as well as point prevalence quit rates by calendar year, were calculated. The question "do you currently use tobacco products?" was used to determine participants' tobacco use status at each follow-up. Each missing follow-up was assumed to be a failure to quit and was included in the quit rate calculation. In other words, the number of total participants recruited was the denominator, while the number of participants who were abstinent at each follow-up comprised the numerators for the point prevalence quit rates. In addition, responses to this question were used to categorize participants' quitting patterns and transitions. We defined 4 quitting patterns: immediate quitters (IQ), delayed quitters (DQ), once quitters (OQ), and never quitters (NQ)..$^{14,15}$ Immediate quitters were those who achieved initial abstinence by 1 month and remained abstinent at the subsequent 2 follow-ups. Delayed quitters were those who did not successfully quit at 1 month or at both 1 month and 3 months but eventually succeeded at 6 months. The term once quitters was used to characterize those who quit smoking at either 1 month or 3 months but relapsed by 6 months. Never quitters were those who failed to be abstinent at any of the 3 follow-ups. Participants were also categorized by their behavioral transitions in tobacco use. Transitions were classified as no transition, forward transition, backward transition, or fluctuation (Figure 1). Staying abstinent or using tobacco across the 3 follow-ups was defined as no transition. Moving from tobacco use to abstinence only once during the 3 follow-ups was considered as forward transition, while the opposite was defined as backward transition. Lastly, transitioning between abstinence and tobacco use twice was considered as fluctuation. The defined quitting patterns could be seen as a further categorization of successful quitters and nonquitters. Those who succeeded at 6 months were further classified as IQ and DQ, whereas those who failed at the end of the program were divided into OQ and NQ.

Based on the findings from our literature review of predictors of successful abstinence, and the information collected from the intake questionnaires regarding patient characteristics, we included age, gender, ethnicity, education, health insurance coverage, quit attempts in the past year, confidence to quit, importance to quit, home ban of smoking, presence of other smokers in household, level of nicotine dependence, tobacco use, and pharmacotherapy use as potential predictors for quitting patterns in the statistical analyses. We categorized age, education, tobacco use in the past 30 days, pharmacotherapy use, importance to quit, confidence to quit, and number of class sessions attended into groups as shown in Table 1. Both confidence to quit and importance to quit were measured on a scale of 1 to 5 with 5 being the most confident/important. We integrated a question regarding whether a patient attempted to quit in the past year with another question about how long the 
Quitting Patterns and Predictors of Success Among Participants in a

Tobacco Cessation Program Provided by Pharmacists in New Mexico

\begin{tabular}{|c|c|c|c|c|c|}
\hline Characteristics & $\mathrm{N}$ & $(\%)$ & Characteristics & $\mathrm{N}$ & $(\%)$ \\
\hline \multicolumn{3}{|l|}{ Age } & \multicolumn{3}{|l|}{ Modified Fagerstrom score } \\
\hline $18-34$ years & 312 & $(21.7)$ & 0-2 (low dependence) & 329 & $(22.9)$ \\
\hline $35-44$ years & 295 & $(20.5)$ & 3-4 (medium dependence) & 680 & $(47.3)$ \\
\hline $45-54$ years & 374 & $(26.0)$ & 5-6 (high dependence) & 184 & $(12.8)$ \\
\hline $55-64$ years & 263 & $(18.3)$ & Not specified & 244 & $(17.0)$ \\
\hline$\geq 65$ years & 89 & $\begin{array}{ll}(6.2) \\
(72)\end{array}$ & \multicolumn{3}{|l|}{ Pharmacotherapy used } \\
\hline Not specified ${ }^{\mathrm{b}}$ & 104 & $(7.2)$ & None & 249 & $(17.3)$ \\
\hline \multicolumn{3}{|l|}{ Gender } & Only nicotine replacement therapy & 552 & $(38.4)$ \\
\hline Female & 729 & $(50.7)$ & Only varenicline & 441 & $(30.7)$ \\
\hline Not specified & 122 & $(8.5)$ & Other combinations & 69 & $(4.8)$ \\
\hline \multicolumn{3}{|l|}{ Ethnicity } & Not specified & 126 & $(8.8)$ \\
\hline White & 934 & $(65.0)$ & \multicolumn{3}{|l|}{ Quit attempts in the past year } \\
\hline Hispanic & 303 & $(21.1)$ & No attempts at all & 332 & $(23.1)$ \\
\hline Other & 77 & $(5.4)$ & Attempted and stayed quit for $\leq 1$ week & 100 & $(7.0)$ \\
\hline Not specified & 123 & $(8.6)$ & Attempted and stayed quit for 1 week & 371 & $(25.8)$ \\
\hline \multicolumn{3}{|l|}{ Education level } & to 1 month & \multirow{3}{*}{200} & \\
\hline Eighth grade or less & 55 & (3.8) & \multirow{2}{*}{$\begin{array}{l}\text { Attempted and stayed quit for } 1 \text { month } \\
\text { to } 6 \text { months }\end{array}$} & & \multirow[t]{2}{*}{$(13.9)$} \\
\hline Some high school or high school & 623 & $(43.4)$ & & & \\
\hline Beyond high school & 613 & $(42.7)$ & \multirow{2}{*}{$\begin{array}{l}\text { Attempted and stayed quit } \\
\text { for }>6 \text { months }\end{array}$} & \multirow[t]{2}{*}{238} & \multirow[t]{2}{*}{$(16.6)$} \\
\hline Not specified & 146 & $(10.2)$ & & & \\
\hline \multicolumn{3}{|l|}{ Health insurance } & Not specified & 196 & (13.6) \\
\hline Yes & 435 & $(30.3)$ & \multicolumn{3}{|l|}{ Allowing smoking at home } \\
\hline No & 768 & $(53.4)$ & Yes, anywhere & 605 & $(42.1)$ \\
\hline \multicolumn{3}{|l|}{ Use tobacco every day } & Only in certain rooms and outdoors & 168 & $(11.7)$ \\
\hline $\begin{array}{l}\text { Use tobacco every day } \\
\text { Yes }\end{array}$ & 1,277 & $(88.9)$ & Outdoors only & 378 & $(26.3)$ \\
\hline No & 49 & $\frac{(00.9)}{(3.4)}$ & No, not allowed at all & 169 & $(11.8)$ \\
\hline Not specified & 111 & $(7.7)$ & Not specified & 117 & $(8.1)$ \\
\hline \multicolumn{3}{|c|}{ Tobacco use pattern in the past 30 days } & \multicolumn{3}{|c|}{ Presence of other tobacco users in the household } \\
\hline Only cigarettes & 740 & $(51.5)$ & Yes, someone who smokes & 564 & $(39.3)$ \\
\hline Cigarettes, pipes, and chew/dips & 476 & $(33.1)$ & Yes, someone who uses smokeless tobacco & 35 & $(2.4)$ \\
\hline Other combinations & 115 & $(8.0)$ & No & 719 & $(50.0)$ \\
\hline Not specified & 106 & $(7.4)$ & Not specified & 119 & $(8.3)$ \\
\hline \multicolumn{3}{|c|}{ Number of cigarettes consumed per day } & \multicolumn{3}{|l|}{ Confidence to quit (scale $1-5$ ) } \\
\hline$\leq$ half pack & 331 & $(23.0)$ & \multicolumn{3}{|l|}{$<2$ (low ronfidence) } \\
\hline$>$ half pack, but $\leq 1$ pack & 606 & $(42.2)$ & 3 & 348 & $(24.2)$ \\
\hline$>1$ pack, but $\leq 1.5$ packs & 175 & $(12.2)$ & 4 & 353 & $(24.6)$ \\
\hline $\begin{array}{l}>1.5 \text { packs } \\
\text { Not specified }\end{array}$ & $\begin{array}{r}92 \\
233\end{array}$ & $\begin{array}{r}(6.4) \\
(16.2)\end{array}$ & 5 (high confidence) & 403 & $(28.0)$ \\
\hline \multicolumn{3}{|c|}{ Time to first use of tobacco after wake up } & Not specified & 114 & $(7.9)$ \\
\hline Within 5 minutes & 585 & $(40.7)$ & \multicolumn{3}{|l|}{ Importance to quit (scale 1-5) } \\
\hline 6-30 minutes & 457 & (31.8) & $\leq 3$ (low importance) & 73 & $(5.1)$ \\
\hline $31-60$ minutes & 178 & $(12.4)$ & 4 & 171 & $(11.9)$ \\
\hline After 60 minutes & 101 & $(7.0)$ & 5 (high importance) & 1,084 & $(75.4)$ \\
\hline Not specified & 116 & $(8.0)$ & Not specified & 109 & $(7.6)$ \\
\hline
\end{tabular}

$a_{N}=1,437$

${ }^{b}$ Not specified = participants who did not respond to certain questions.

NMPCF $=$ New Mexico Pharmaceutical Care Foundation.

patient remained abstinent at the latest attempt into 1 variable to represent his/her quitting attempt in the past year. The variable consisted of 5 groups: "no attempts at all," "attempted and stayed quit for 1 week or less," "attempted and stayed quit for
1 week to 1 month," "attempted and stayed quit for 1 month to 6 months," and "attempted and stayed quit for more than 6 months." We kept the original categorizations from the intake questionnaire for the other potential predictors. 


\section{FIGURE 2 Point Prevalence Quit Rates by Calendar Year at 1 Month, 3 Months, and 6 Months}

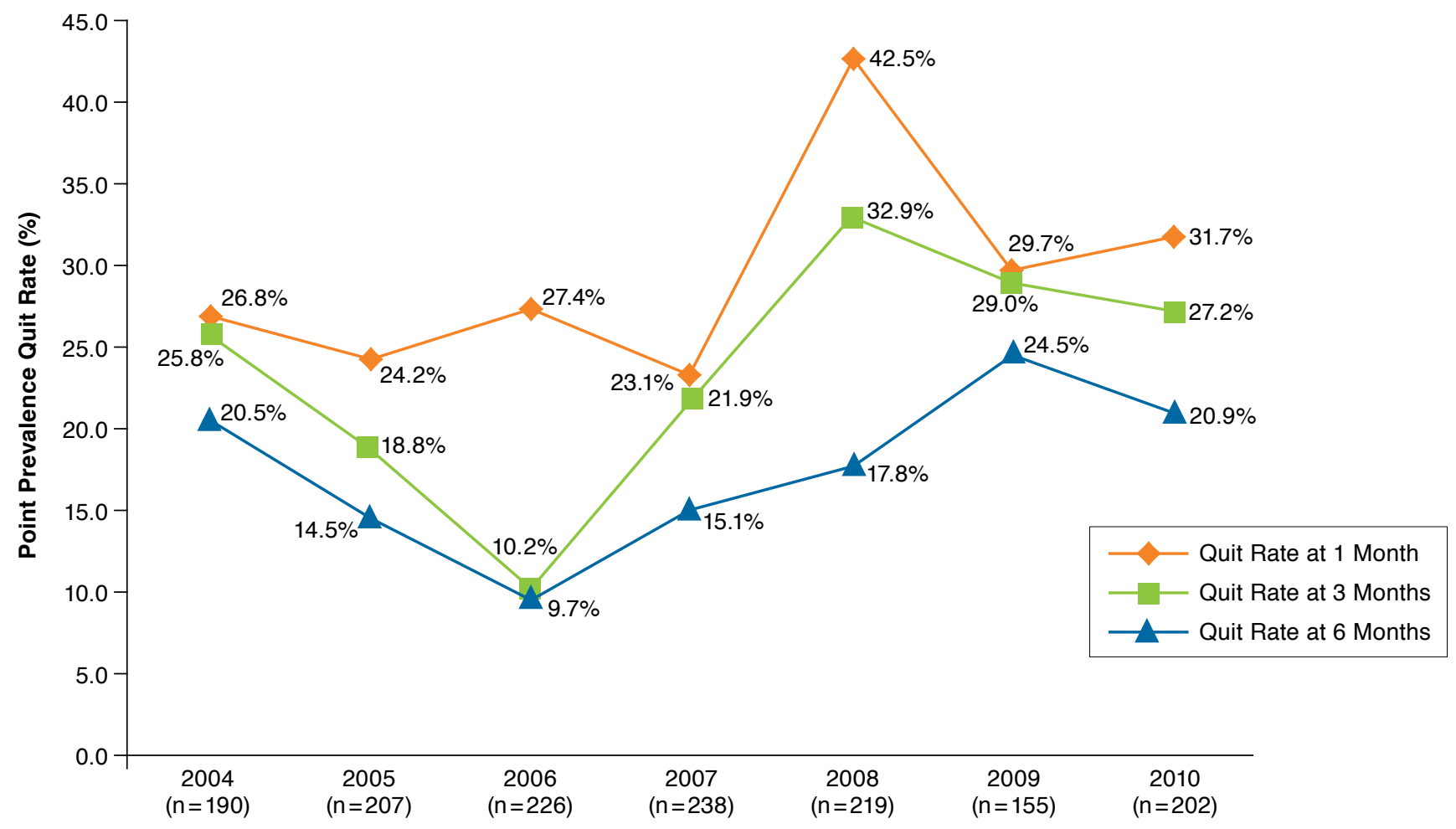

Although the Fagerstrom Test for Nicotine Dependence (FTND), a widely used instrument for measuring nicotine dependence, was not administered to participants, the intake questionnaire adopted 2 questions from the FTND to evaluate participants' level of nicotine dependence: (1) "how many cigarettes (or cigars, bowls of pipes, etc.)/day do you smoke?" and (2) "how soon after you wake up do you first use tobacco?" In the FTND, number of cigarettes per day was scored on a scale of 0 to 3, with 0.5 pack per day or less being $0 ; 0.5$ pack to 1 pack being 1 ; 1 pack to 1.5 packs being 2 ; and more than 1.5 packs being 3 . Time to first use of tobacco after waking up was also scored on a 0-3 scale, with "within 5 minutes" being 3; "6-30 minutes" being 2; "31-60 minutes" being 1 ; and "after 60 minutes" being 0 . We adopted the same scoring scheme and created a modified FTND score by summing up the individual scores from the 2 questions. The modified FTND score had possible values ranging from 0 to 6 , with higher scores indicating higher nicotine dependence, and was categorized into 4 groups based on percentile distribution including score 0-2, score 3, score 4, and score 5-6.

Chi-square tests were used to compare the point prevalence quit rates across years. Multinomial logistic regressions were performed for characterizing quitting patterns. The potential predictors displayed in Table 1 were first examined individu- ally in univariate models. All variables with resulting $P$ values less than or equal to 0.2 were included in the multivariate analysis. The backward elimination strategy was adopted for the multivariate analyses. Variables with a type $3 P$ value being greater than 0.2 in the initial full model were tested individually in descending order of $P$ value for their importance to the model. The type 3 test evaluated the significance of an individual variable after controlling for other variables in the model. ${ }^{19}$ The likelihood ratio (LR) test was used to compare the full model with the reduced model. The decision to keep a variable in the model was made based on the statistical significance of the LR test. SAS 9.2 (Cary, NC) was used to perform all the statistical analyses. This study was reviewed by the University of New Mexico's institutional review board and received expedited approval.

\section{Results}

After excluding those who failed to respond to any of the 4 interviews, 1,437 participants were included for analysis. Table 1 summarizes baseline characteristics of the study sample. The majority of the participants were white (70.1\%), under aged 65 years (93\%), and did not have health insurance $(64 \%)$. Cigarettes were the most common single nicotine product used (55.6\%), but $35.9 \%$ used multiple nicotine 
Quitting Patterns and Predictors of Success Among Participants in a

Tobacco Cessation Program Provided by Pharmacists in New Mexico

\begin{tabular}{|c|c|c|}
\hline $\begin{aligned} & \text { TABLE 2 } \text { Quitting Pat } \\
& \text { Included Pa } \\
& \text { Tobacco Ce }\end{aligned}$ & \multicolumn{2}{|c|}{$\begin{array}{l}\text { Quitting Patterns and Transitions of } \\
\text { Included Participants from NMPCF } \\
\text { Tobacco Cessation Programa }\end{array}$} \\
\hline Quitting patterns & $\mathbf{N}$ & (\%) \\
\hline Immediate quitters & 145 & $(10.1)$ \\
\hline Delayed quitters & 113 & $(7.9)$ \\
\hline Once quitters & 298 & $(20.7)$ \\
\hline Never quitters & 881 & $(61.3)$ \\
\hline \multicolumn{3}{|l|}{ Quitting transitions } \\
\hline Forward transition & 87 & $(6.1)$ \\
\hline Backward transition & 250 & $(17.4)$ \\
\hline Fluctuation & 74 & $(5.2)$ \\
\hline No transition & 1,026 & $(71.4)$ \\
\hline \multicolumn{3}{|l|}{ Patterns and transitions } \\
\hline Immediate quitters, no transition & 145 & $(10.1)$ \\
\hline Delayed quitters, forward transition & 87 & $(6.1)$ \\
\hline Delayed quitters, fluctuation & 26 & $(1.8)$ \\
\hline Once quitters, fluctuation & 48 & (3.3) \\
\hline Once quitters, backward transition & 250 & $(17.4)$ \\
\hline Never quitters, no transition & 881 & $(61.3)$ \\
\hline
\end{tabular}

products. Around $15.4 \%$ of the participants had a modified FTND score of 5 or 6 , indicating a high level of nicotine dependence. Most of the participants indicated the high levels of importance for them to quit (81.6\% scored 5 on the scale), but $43 \%$ reported their confidence to quit was 3 or less on a scale of 1 (least) to 5 (most).

The average point prevalence quit rate at 1 month, 3 months, and 6 months was 29.3\%, 23.3\%, and 18.0\%, respectively. Point prevalence quit rates by calendar year are presented in Figure 2. The chi-square tests showed that the quit rates were significantly different across years for all 3 follow-ups $(P \leq 0.0002)$. Year 2009 had the highest quit rate at 6 months (24.5\%). Year 2004 and 2010 also achieved a 6-month quit rate greater than $20 \%$. Based on our definition for quitting patterns, the study sample consisted of 145 (10.1\%) IQs, 113 (7.9\%) DQs, 298 (20.7\%) OQs, and 881 (61.3\%) NQs. As for their transitions of tobacco use status during the program period, 87 (6.1\%) moved forward from using tobacco to abstinence; 250 (17.4\%) had a backward transition; 74 (5.2\%) participants' abstinence status fluctuated; and 1,026 (71.4\%) participants had the same status, staying abstinent, or using tobacco across 3 follow-ups (Table 2).

The results of the univariate analysis showed that gender, ethnicity, education level, daily use of tobacco, tobacco use pattern in the past 30 days, home ban of smoking, modified FTND scores, pharmacotherapy used, importance to quit, and confidence to quit were associated with quitting patterns. Therefore, those variables were selected into the full multivariate multinomial logistic regression model. The type $3 P$ value for ethnicity and importance to quit were greater than 0.2 in the full model, and the LR test was performed to examine the importance of these 2 predictors in the model. The LR test indicated that removing ethnicity statistically significantly impacted the model $(P<0.001)$, while the influence of deleting importance to quit on the model was statistically nonsignificant $(P=0.38)$. Consequently, we kept ethnicity in the model but removed importance to quit.

After controlling for all the other variables in the model, participants with an education level of eighth grade or less were more likely to be IQ than those with some or complete high school education (odds ratio $[\mathrm{OR}]=4.21,95 \%$ confidence interval [CI]: 1.66, 10.70). Participants who used cigarettes, pipes, and chew/dips in the past 30 days were more likely to be DQ (OR $=2.19$ 95\% CI: $1.30,3.68)$ and OQ (OR $=1.80,95 \%$ CI: $1.27,2.57)$ compared with those who only smoked cigarettes. Although the modified FTND score was not significantly associated with quitting patterns across groups, a trend in reduced likelihood of quitting was observed among those with higher nicotine dependence. Patients who used nicotine replacement therapy $(\mathrm{OR}=2.16,95 \% \mathrm{CI}: 1.31,3.56)$ or varenicline $(\mathrm{OR}=2.04,1.21,95 \% \mathrm{CI}: 3.43)$ were more likely to be OQ than those who did not use any forms of pharmacotherapy. This finding suggested that pharmacotherapies were helpful with respect to achieving short-term success, but they alone might not be sufficient for maintaining long-term abstinence. Home ban of smoking was inversely related to the likelihood of successful quitting after adjusting for other variables. Participants with higher confidence levels were more likely to be IQ and OQ than those with lower confidence levels (Table 3). Associations between participant characteristics and success at 6 months were also evaluated. The findings are compatible with the pattern analysis (Appendix, available in online article).

\section{Discussion}

$\overline{\text { Although tobacco use has been recognized as a health hazard }}$ for many years, it remains a major public health concern in the United States. Tobacco use is one of the Healthy People 2020 topic areas retained from Healthy People 2010. Different from Healthy People 2010, 2 new objectives have been added to Healthy People 2020, namely increasing tobacco screening in health care settings and tobacco cessation counseling in health care settings by $10 \% .{ }^{20}$ Considering that attributable deaths to tobacco use were almost equal to deaths caused by cerebrovascular disease, the number 2 killer, reducing tobacco use is a pressing task for the United States. ${ }^{21}$ A pharmacist-assisted tobacco cessation service in the United Kingdom achieved a quit rate of $3.6 \%$ at 52 weeks. An economic evaluation showed that the service was cost-effective despite the relatively low quit rate. ${ }^{22}$ The reported incremental cost per quality adjusted life year gained for one-on-one pharmacist counseling was 2,600 euros, which is only one-tenth of the cost-effectiveness threshold set by the National Institute for Health and Clinical Excellence. $^{23}$ 
Quitting Patterns and Predictors of Success Among Participants in a

Tobacco Cessation Program Provided by Pharmacists in New Mexico

TABLE 3 Multivariate Analysis-Characteristics of Immediate Quitters, Delayed Quitters, and Once Quitters Compared with Never Quitters

\begin{tabular}{|c|c|c|c|c|c|c|}
\hline & \multicolumn{2}{|c|}{$\begin{array}{c}\text { Immediate Quitters vs. } \\
\text { Never Quitters (OR, } 95 \% \text { CI) }\end{array}$} & \multicolumn{2}{|c|}{$\begin{array}{c}\text { Delayed Quitters vs. } \\
\text { Never Quitters (OR, 95\% CI)a }\end{array}$} & \multicolumn{2}{|c|}{$\begin{array}{c}\text { Once Quitters vs. } \\
\text { Never Quitters }(\mathrm{OR}, 95 \% \mathrm{CI})^{\mathrm{a}} \\
\end{array}$} \\
\hline \multicolumn{7}{|l|}{ Gender (reference: female) } \\
\hline Male & 0.88 & $(0.58,1.35)$ & 1.61 & $(0.99,2.62)$ & 0.85 & $(0.61,1.18)$ \\
\hline \multicolumn{7}{|l|}{ Ethnicity (reference: white) } \\
\hline Hispanic & 1.20 & $(0.73,1.96)$ & 0.78 & $(0.43,1.42)$ & 0.90 & $(0.61,1.33)$ \\
\hline Other & 1.13 & $(0.46,2.74)$ & 1.22 & $(0.49,3.00)$ & 1.09 & $(0.56,2.11)$ \\
\hline \multicolumn{7}{|c|}{ Education (reference: some/complete high school) } \\
\hline Eighth grade or less & 4.21 & $(1.66,10.70)$ & 2.08 & $(0.68,6.36)$ & 1.98 & $(0.89,4.40)$ \\
\hline Beyond high school & 1.22 & $(0.79,1.90)$ & 0.93 & $(0.56,1.53)$ & 0.84 & $(0.60,1.17)$ \\
\hline \multicolumn{7}{|l|}{ Use tobacco daily (reference: no) } \\
\hline Yes & 0.83 & $(0.17,4.02)$ & 0.32 & $(0.11,0.96)$ & 0.52 & $(0.20,1.33)$ \\
\hline \multicolumn{7}{|c|}{ Tobacco use in the past 30 days (reference: only cigarettes) } \\
\hline Cigarettes, pipes, and chew/dips & 1.38 & $(0.87,2.21)$ & 2.19 & $(1.30,3.68)$ & 1.80 & $(1.27,2.57)$ \\
\hline Other & 0.91 & $(0.35,2.35)$ & 1.10 & $(0.39,3.12)$ & 1.61 & $(0.85,3.03)$ \\
\hline \multicolumn{7}{|c|}{ Modified Fagerstrom score (reference: $0-2$ ) } \\
\hline 3 & 0.66 & $(0.38,1.15)$ & 0.87 & $(0.49,1.54)$ & 0.69 & $(0.45,1.07)$ \\
\hline 4 & 0.64 & $(0.36,1.13)$ & 0.39 & $(0.19,0.79)$ & 0.78 & $(0.51,1.21)$ \\
\hline 5-6 high dependence & 0.78 & $(0.39,1.54)$ & 0.57 & $(0.25,1.29)$ & 0.80 & $(0.47,1.36)$ \\
\hline \multicolumn{7}{|l|}{ Pharmacotherapy used (reference: none) } \\
\hline Only NRT & 0.71 & $(0.39,1.28)$ & 1.40 & $(0.70,2.80)$ & 2.16 & $(1.31,3.56)$ \\
\hline Only varenicline & 1.38 & $(0.78,2.46)$ & 1.14 & $(0.55,2.38)$ & 2.04 & $(1.21,3.43)$ \\
\hline Other & 1.36 & $(0.55,3.36)$ & 1.25 & $(0.37,4.17)$ & 1.44 & $(0.59,3.53)$ \\
\hline \multicolumn{7}{|c|}{ Allowing smoking at home (reference: yes, anywhere) } \\
\hline Only in certain rooms and outdoors & 0.22 & $(0.09,0.54)$ & 0.90 & $(0.44,1.84)$ & 0.61 & $(0.37,1.01)$ \\
\hline Outdoors only & 0.63 & $(0.39,1.03)$ & 0.65 & $(0.36,1.18)$ & 0.65 & $(0.44,0.96)$ \\
\hline No, not allowed at all & 0.44 & $(0.20,0.95)$ & 0.68 & $(0.31,1.50)$ & 0.64 & $(0.37,1.11)$ \\
\hline \multicolumn{7}{|l|}{ Confidence to quit (reference: $<3$ ) } \\
\hline 3 & 1.95 & $(0.93,4.08)$ & 0.83 & $(0.36,1.94)$ & 1.08 & $(0.63,1.88)$ \\
\hline 4 & 2.58 & $(1.23,5.44)$ & 1.45 & $(0.65,3.25)$ & 1.75 & $(1.03,3.00)$ \\
\hline 5 high confidence & 2.43 & $(1.14,5.15)$ & 1.89 & $(0.87,4.12)$ & 2.16 & $(1.27,3.67)$ \\
\hline
\end{tabular}

Compared with the quit rates of previous pharmacist-assisted programs or programs led by other health professionals in the United States (range: 9.9\%-26.0\%), the average quit rates achieved by the NMPCF program are similar, despite our conservative calculation approach (Figure 2) ${ }^{7,9,24}$ The point prevalence quit rates demonstrate the overall trends of the program. Specifically, as expected, the highest quit rate each year was at 1 month, followed by 3 months, and then 6 months. Also, other than the dip in 2006 and a peak in 2008, the rates appear to improve slightly each year. This may have been due to unsuccessful pharmacists leaving the NMPCF program or improved counseling techniques gained by experience among pharmacists.

The results of the pattern analysis demonstrated the existence of different quitting patterns among tobacco users. Participants were likely to be at different stages of quitting tobacco when recruited, spreading among precontemplation, contemplation, and preparation. ${ }^{25,26}$ In applying the TTM to our findings, we assumed that those who achieved immediate success (IQ) were possibly in preparation at recruitment and were ready to make a change. Those who were in precontemplation or contemplation needed time and help from pharmacists before taking the action to quit and thus were more likely to be DQ and OQ. Our finding about pharmacotherapy aids is consistent with the literature, which indicates medication and counseling combined increases the odds of abstinence. ${ }^{10}$

Our study provides valuable information and presents strengths over previous studies. Compared with other pharmacist-assisted tobacco cessation programs reported in the literature, our study sample, consisting of 1,437 participants residing in rural or urban areas throughout New Mexico and 7 years in duration, is unprecedentedly large. Compared with tobacco cessation programs led by other health care professionals, the program quit rates demonstrated that having pharmacists deliver tobacco cessation services results in similar success rates. Moreover, our findings in quitting patterns increased our 
knowledge of the process of quitting tobacco, which is a critical but understudied dimension of tobacco cessation. Future research is needed for studying quitting patterns in more detail and identifying additional explanations for the existence of different quitting patterns. Longitudinal studies that frequently follow up with participants regarding their tobacco use status will be informative in understanding the timing, directions, and frequency of behavioral transitions among tobacco users and characterizing quitting patterns in more detail. In addition, future studies should consider collecting data beyond sociodemographic characteristics. There are very few studies that have evaluated the relationship between type of tobacco and likelihood of abstinence. Our finding that participants who use cigarettes, pipes, and chew/dips are more likely to be DQs and OQs needs to be verified. Information on health conditions, social support, readiness to quit, type of tobacco, and intervention characteristics such as program intensity will help researchers better explain the existence of different quitting patterns and enable clinicians to more precisely target participants with appropriate interventions.

The annual TUPAC grant included funding for education programs for the pharmacists and for foundation/association administration staff. Direct cessation funding was $\$ 200$ per participant for the pharmacist counseling component and $\$ 137.50$ for medications for the first month. The program provided additional funding for medications in later months for a proportion of participants at the specific request of a pharmacist based on recommendation and analysis of participant need.

\section{Limitations}

Some of the results from the pattern analysis are not clearly intuitive. The finding on home ban of smoking contradicts existing literature, ${ }^{27-29}$ but this is not necessarily implausible. Since social support is an important predictor of success in quitting tobacco, being able to smoke at home might be an indicator of a harmonious domestic relationship or strong family support. ${ }^{30,31}$ Another potential explanation is that those participants without a home ban are more determined to quit. Their decision to quit comes from themselves rather than externally from family members. The training stressed that personal commitment to quitting is the most important determinant of successful abstinence. Importance to quit was unrelated to abstinence. One reason is that there was little variation in this measure ( $82 \%$ scored the highest possible value). However, another potential explanation is that participants who scored high on this measure might have health conditions that forced them to quit but were not ready to quit. Unfortunately, information regarding health conditions was not collected. We acknowledge this as a limitation of the study. Participants with eighth grade or less education were found to have increased odds of quitting immediately than others with higher education. However, the estimated OR was imprecise with a wide CI $(\mathrm{OR}=4.21,95 \% \mathrm{CI}$ : 1.66-10.70) due to the limited number of individuals $(n=55$, $3.8 \%$ ) in the category of eighth grade or less.
Another limitation of this study is that nearly $50 \%$ of the information on participants' tobacco use status at each of the 3 follow-ups was missing due to loss to follow-up. We presumed that the missing data were mainly due to participants' lack of success and counted it as a failure. The pharmacists agreed that loss to follow-up was most likely due to relapse. We note that the primary goal of the NMPCF TUBAC was to improve public health. Since it was not designed specifically for conducting research, data collection was not a priority. Follow-up data were not routinely audited during the conduct of NM TUPAC. Smoking status at follow-ups was self-reported rather than biochemically verified. We also note that point prevalence quit rates may be overestimated. As shown in a systematic review on the relationship between self-reported and cotinine-assessed smoking status, self-report may overestimate abstinence rates between $3 \%$ to $22 \%$ when being compared with saliva cotinine assessment. ${ }^{32}$ Another limitation is that the modified FTND instrument has not been validated.

\section{Conclusions}

Our results show that having community pharmacists provide smoking cessation interventions resulted in quitting success rates similar to other health professionals. ${ }^{10}$ Since pharmacists are a widely available resource for their patients, managed care organizations may be able to improve the health, and avoid subsequent tobacco-related adverse health outcomes, of their enrollees by implementing a program similar to the NMPCF Tobacco Cessation Program. Such a program includes education for pharmacists, therapeutic protocols regarding use of tobacco cessation products, regular counseling and follow-up, and adequate reimbursement for tobacco cessation services. Furthermore, data collection regarding the participants enrolled and achievement of tobacco cessation is a critical component for evaluation of outcomes. The multivariate analysis of quitting patterns provides insights into which individuals may have more difficulty achieving tobacco cessation and thus may need more intensive interventions (Table 3). Another strategy may be to focus efforts among those most likely to be IQs or DQs. Specifically, those with higher confidence to quit, use tobacco intermittently, had Fagerstrom scores of 4 , and those using multiple tobacco products had more success in the NMPCF tobacco cessation program. We did not find a consistent pattern associated with use of tobacco cessation products (replacement or nicotine receptor blockers); however, this is likely because the vast majority of participants (80.9\%) used some type of pharmaceutical product to assist in smoking cessation.

Our findings have implications related to TTM and may be helpful in designing tobacco cessation programs in managed care or community pharmacies. Specifically, programs might focus on individuals who use multiple tobacco products, include educational programs to increase confidence for success, involve family members, and incorporate guidelinedriven prescribing of tobacco cessation products. 


\section{Authors}

XIAN SHEN, MS, is Research Assistant and PhD Candidate, University of Maryland Baltimore School of Pharmacy, Baltimore, Maryland. AMY BACHYRYCZ, PharmD, is Teaching Faculty, University of New Mexico College of Pharmacy, and Pharmacist, Walgreens Patient Care Center, Albuquerque, New Mexico. JOE R. ANDERSON, PharmD, is Associate Professor, and DENNIS W. RAISCH, PhD, MS, RPh, is Professor, University of New Mexico College of Pharmacy, Albuquerque, New Mexico. DALE TINKER, $B A$, is Treasurer, New Mexico Pharmaceutical Care Foundation, and Executive Director, New Mexico Pharmacists Association, Albuquerque, New Mexico.

AUTHOR CORRESPONDENCE: Dennis W. Raisch, PhD, MS, RPh, Professor and Chair, Graduate Program in Pharmacoeconomics, Epidemiology, Pharmaceutical Policy and Outcomes Research, College of Pharmacy, University of New Mexico, 1 University of New Mexico, MSC 09 5360, Albuquerque, NM 87131.

Tel.: 505.272.2130; Fax: 505.272.6749; E-mail: draisch@salud.unm.edu.

\section{DISCLOSURES}

The authors report no conflicts of interest.

Study concept and design were primarily contributed by Shen, with assistance from Raisch. Data collection was primarily performed by Tinker, with assistance from Bachyrycz; all authors contributed equally to data interpretation. The manuscript was written primarily by Shen, with assistance from Raisch, and revised by Shen, Tinker, and Raisch.

\section{REFERENCES}

1. DiClemente CC, Prochaska JO. Self-change and therapy change of smoking behavior: a comparison of processes of change in cessation and maintenance. Addict Behav. 1982;7(2):133-42.

2. Prochaska JO, DiClemente CC. Stages and processes of self-change of smoking: toward an integrative model of change. Journal Consult Clin Psychol. 1983;51(3):390-95

3. Cahill K, Lancaster T, Green N. Stage-based interventions for smoking cessation. Cochrane Database Syst Rev. 2010(11):CD004492.

4. Pisinger $C$, Aadahl M, Toft U, Jørgensen T. Motives to quit smoking and reasons to relapse differ by socioeconomic status. Prev Med. 2011;52(1):48-52.

5. Yang T, Fisher KJ, Li F, Danaher BG. Attitudes to smoking cessation and triggers to relapse among Chinese male smokers. BMC Public Health. 2006;6:65.

6. Dent LA, Scott JG, Lewis E. Pharmacist-managed tobacco cessation program in Veterans Health Administration community-based outpatient clinic. J Am Pharm Assoc (2003). 2004:44(6):700-14.

7. Roth MT, Westman EC. Use of bupropion SR in a pharmacist-managed outpatient smoking-cessation program. Pharmacotherapy. 2001;21(5):636-41. 8. Zillich AJ, Ryan M, Adams A, Yeager B, Farris K. Effectiveness of a pharmacist-based smoking-cessation program and its impact on quality of life. Pharmacotherapy. 2002;22(6):759-65.

9. Kennedy DT, Giles JT, Chang ZG, Small RE, Edwards JH. Results of a smoking cessation clinic in community pharmacy practice. J Am Pharm Assoc (Wash). 2002;42(1):51-56.

10. Fiore MC, Jaén CR, Baker TB, et al. Treating tobacco use and dependence: 2008 update. Clinical Practice Guideline. Rockville, MD: U.S. Department of Health and Human Services. Public Health Service. May 2008. Available at: http://bphc.hrsa.gov/buckets/treatingtobacco.pdf. Accessed April 30, 2014

11. Unrod M, Smith M, Spring B, DePue J, Redd W, Winkel G. Randomized controlled trial of a computer-based, tailored intervention to increase smoking cessation counseling by primary care physicians. J Gen Intern Med. 2007;22(4):478-84.
12. Wewers ME, Ferketich AK, Harness J, Paskett ED. Effectiveness of a nurse-managed, lay-led tobacco cessation intervention among Ohio Appalachian women. Cancer Epidemiol Biomarkers Prev. 2009;18(12):3451-58. 13. Liberman JN, Lichtenfeld MJ, Galaznik A, et al. Adherence to varenicline and associated smoking cessation in a community-based patient setting. J Manag Care Pharm. 2013;19(2):125-31. Available at: http://www.amcp.org/ WorkArea/DownloadAsset.aspx?id=16219.

14. Gonzales D, Jorenby DE, Brandon TH, Arteaga C, Lee TC. Immediate versus delayed quitting and rates of relapse among smokers treated successfully with varenicline, bupropion SR or placebo. Addiction. 2010;105(11):2002-13.

15. Hajek P, Tonnesen P, Arteaga C, Russ C, Tonstad S. Varenicline in prevention of relapse to smoking: effect of quit pattern on response to extended treatment. Addiction. 2009;104(9):1597-602.

16. University of California San Francisco. Rx for Change. Clinician-assisted tobacco cessation. Available at: http://rxforchange.ucsf.edu/. Accessed April 24, 2014.

17. Williams DM. Preparing pharmacy students and pharmacists to provide tobacco cessation counselling. Drug Alcohol Rev. 2009;28(5):533-40.

18. Glynn TJ, Manley MW. How to help your patients stop smoking: a National Cancer Institute manual for physicians. NIH Publication No. 89-3064. Bethesda, MD: National Institutes of Health; 1997.

19. SAS Institute. Type III tests. SAS OnlineDoc V8. Carey, NC. Available at: http://v8doc.sas.com/sashtml/insight/chap39/sect25.htm. Accessed April 24, 2014 .

20. Healthy People 2020. 2020 topics and objectives: tobacco use. Healthy People.gov. August 2013. Available at: http://www.healthypeople.gov/2020/topicsobjectives2020/objectiveslist.aspx?topicId=41. Accessed April 24, 2014. 21. World Health Organization. World health statistics 2008. Available at: http://www.who.int/gho/publications/world_health_statistics/EN_WHS08_ Full.pdf. Accessed April 24, 2014.

22. Bauld L, Boyd KA, Briggs AH, et al. One-year outcomes and a costeffectiveness analysis for smokers accessing group-based and pharmacy-led cessation services. Nicotine Tob Res. 2011;13(2):135-45.

23. Appleby J, Devlin N, Parkin D. NICE's cost effectiveness threshold. BMJ. 2007;335(7616):358-59.

24. Lancaster T, Dobbie W, Vos K, Yudkin P, Murphy M, Fowler G. Randomized trial of nurse-assisted strategies for smoking cessation in primary care. Brit J Gen Pract. 1999;49(440):191-94.

25. Kohler CL, Schoenberger YM, Tseng TS, Ross L. Correlates of transitions in stage of change for quitting among adolescent smokers. Addict Behav. 2008;33(12):1615-18

26. DiClemente CC, Delahanty JC, Kofeldt MG, Dixon L, Goldberg R, Lucksted A. Stage movement following a 5A's intervention in tobacco dependent individuals with serious mental illness (SMI). Addict Behav. 2011;36(3):261-64.

27. Fu SS, Burgess DJ, van Ryn M, et al. Smoking-cessation strategies for American Indians: should smoking-cessation treatment include a prescription for a complete home smoking ban? Am J Prev Med. 2010;39(6 Suppl 1):S56-S65. 28. Ji M, Hofstetter CR, Hovell M, et al. Smoking cessation patterns and predictors among adult Californians of Korean descent. Nicotine Tob Res. 2005;7(1):59-69.

29. Hyland A, Higbee C, Travers MJ, et al. Smoke-free homes and smoking cessation and relapse in a longitudinal population of adults. Nicotine Tob Res. 2009;11(6):614-18.

30. Rice VH, Templin T, Fox DH, et al. Social context variables as predictors of smoking cessation. Tob Control. 1996;5(4):280-85.

31. Lawhon D, Humfleet GL, Hall SM, Munoz RF, Reus VI. Longitudinal analysis of abstinence-specific social support and smoking cessation. Health Psychol. 2009;28(4):465-72.

32. Connor Gorber S, Schofield-Hurwitz S, Hardt J, Levasseur G, Tremblay $\mathrm{M}$. The accuracy of self-reported smoking: a systematic review of the relationship between self-reported and cotinine-assessed smoking status. Nicotine Tob Res. 2009;11(1):12-24. 
Quitting Patterns and Predictors of Success Among Participants in a

Tobacco Cessation Program Provided by Pharmacists in New Mexico

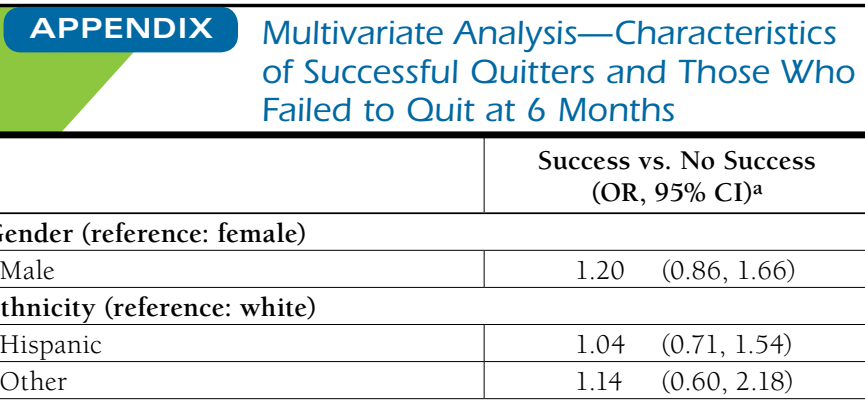

Education (reference: some/complete high school)

\begin{tabular}{l|rl}
\hline Eighth grade or less & 2.32 & $(1.14,4.74)$ \\
\hline Beyond high school & 1.15 & $(0.82,1.61)$ \\
\hline Use tobacco daily (reference: no) & & \\
\hline Yes & 0.57 & $(0.23,1.36)$ \\
\hline
\end{tabular}

Tobacco use in the past 30 days (reference: only cigarettes)

\begin{tabular}{l|ll}
\hline Cigarettes, pipes, and chew/dips & 1.39 & $(0.98,1.99)$ \\
\hline Other & 0.85 & $(0.42,1.74)$
\end{tabular}

Modified Fagerstrom score (reference: $0-2$ )

\begin{tabular}{l|ll}
\hline 3 & 0.82 & $(0.54,1.23)$ \\
\hline 4 & 0.57 & $(0.36,0.89)$ \\
\hline $5-6$ high dependence & 0.72 & $(0.42,1.23)$ \\
\hline
\end{tabular}

Pharmacotherapy used (reference: none)

\begin{tabular}{l|ll}
\hline Only NRT & 0.81 & $(0.51,1.27)$ \\
\hline Only varenicline & 1.09 & $(0.69,1.74)$ \\
\hline Other & 1.21 & $(0.57,2.54)$ \\
\hline
\end{tabular}

Allowing smoking at home (reference: yes, anywhere)

\begin{tabular}{l|rl}
\hline Only in certain rooms and outdoors & 0.53 & $(0.31,0.92)$ \\
\hline Outdoors only & 0.71 & $(0.48,1.04)$ \\
\hline No, not allowed at all & 0.60 & $(0.34,1.06)$ \\
\hline Confidence to quit (reference: $<3)$ & & \\
\hline 3 & 1.36 & $(0.78,2.39)$ \\
\hline 4 & 1.75 & $(1.01,3.05)$ \\
\hline 5 high confidence & 1.78 & $(1.02,3.10)$ \\
\hline
\end{tabular}

a Significant values $(P<0.05)$, based upon significant confidence intervals, are displayed in bold font.

$\mathrm{CI}=$ confidence interval; $\mathrm{NRT}=$ nicotine replacement therapy; $\mathrm{OR}=$ odds ratio. 